\title{
Numerical aspects of fluid infusion inside a compressible porous medium undergoing large strains
}

\author{
Pierre Celle* - Sylvain Drapier** — Jean-Michel Bergheau*** \\ * ESI France SAS - Parc d'Affaires SILIC \\ 99, rue des Solets, F-94513 Rungis cedex \\ pierre.celle@esi-group.com \\ ** SMS Division and LTDS, UMR 5513 CNRS \\ Ecole Nationale Supérieure des Mines de Saint-Etienne \\ 158, cours Fauriel, F-42023 Saint-Etienne cedex 2 \\ drapier@emse.fr \\ *** LTDS, UMR 5513 CNRS \\ Ecole Nationale d'Ingénieurs de Saint-Etienne \\ 58, rue Jean Parot, F-42023 Saint-Etienne cedex 2 \\ bergheau@enise.fr
}

\begin{abstract}
A new model for a thermo-reactive fluid flow across a highly compressible porous medium has been proposed and employed to predict infusion-based manufacturing processes for polymer composites (Celle, 2006). These techniques consist in mixing the reinforcements and the resin during the manufacturing cycle, transversely to the fabrics plane, by applying a pressure on the resin/preform stacking. This yields cost reductions and avoids filling problems. The introduction of a numerical model in a finite element software to study infusion-based processes will increase their diffusion by a reliable prediction of both part thickness and porosity (Celle et al., 2008). The present paper deals mainly with the numerical treatments related to the resin layer.

RÉSUMÉ. Un nouveau modèle pour l'étude de l'écoulement thermo-réactif d'un fluide à travers un milieu poreux fortement compressible a été développé et appliqué aux procédés d'élaboration des matériaux composites par infusion (Celle, 2006). Ces techniques consistent à réaliser le mélange, à travers leur épaisseur, des nappes de renforts et de la résine, par application d'une pression sur l'empilement nappes/résine. Ceci conduit à une réduction des coûts et évite l'apparition de zones sèches. L'intégration d'un modèle numérique d'infusion dans un code de calcul par éléments finis facilitera la diffusion de ces solutions en apportant une prévision fiable des épaisseurs et des fractions volumiques de fibres (Celle et al., 2008). Dans ce papier, on s'intéresse plus spécifiquement aux traitements numériques associés à la zone de résine pure.
\end{abstract}

KEYWORDS: LRI (Liquid Resin Infusion), RFI (Resin Film Infusion), composites materials, finite element method, porous media, finite strains.

MOTS-CLÉS: LRI (Liquid Resin Infusion), RFI (Resin Film Infusion), matériaux composites, méthode des éléments finis, milieux poreux, grandes déformations.

DOI:10.3166/REMN.17.819-827 (c) 2008 Lavoisier, Paris

REMN $-17 / 2008$. Giens 2007, pages 819 to 827 


\section{Introduction}

Composite direct manufacturing processes consist in mixing reinforcements and resin during the elaboration stage, thus reducing handling and costs. Injection-like processes such as RTM (Resin Transfer Moulding) are now widely used industrially (Figure 1). However, for pieces presenting large section to volume ratios, such that hulls, decks, wing skins... the distances resin has to travel may lead to void formation.

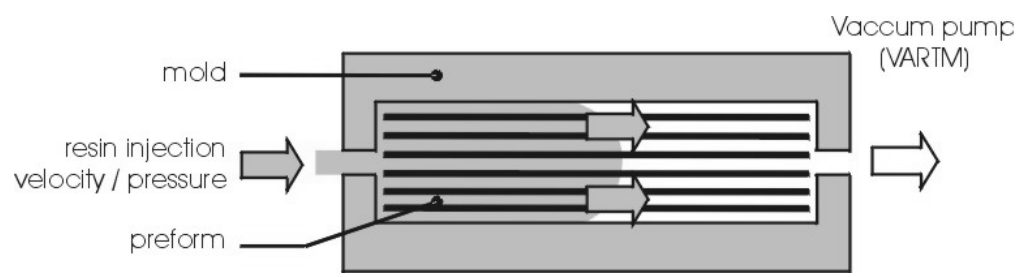

Figure 1. RTM process

On the contrary, infusion processes, such as $L R I$ (Liquid Resin Infusion) and RFI (Resin Film Infusion), permit to avoid such problems since resin-fibre mixing takes place in the direction transverse to the fabric plane (Figure 2). In those processes, preforms are laid up over a solid resin film (RFI Figure 2a) or below a draining fabric ( $L R I$ Figure $2 \mathrm{~b}$ ) in a semi-rigid mould. This stacking may be covered by a punched counter-mould to ensure the top part finish quality. The stacking is then sealed in a vacuum bag so that vacuum can be achieved. Then resin infuses across the preform thickness mainly under the mechanical pressure induced by the atmospheric pressure outside the vacuum bag. A further assistance can consist in applying air depression to vents and/or prescribe an additional external pressure.

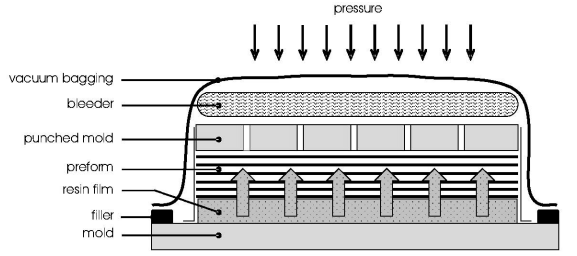

(a)

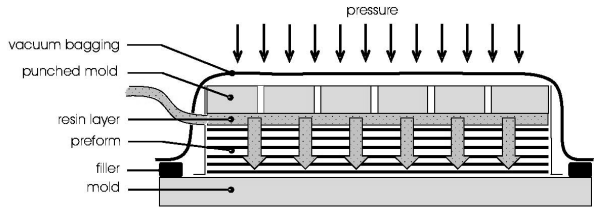

(b)

Figure 2. Infusion manufacturing processes: (a) RFI, (b) LRI 
These promising techniques permit to improve the quality of the final parts while reducing cycle times and costs. However, thicknesses and fibre volume fractions are still poorly controlled. At the moment, pre-industrial phases and developments rely mainly on prototyping and trial-error stages, both quite expensive. A numerical model of this complex hydro-mechanical problem, coupled with thermo-chemicophysics, has been developed and implemented based on the eulerian libraries PROFLOT ${ }^{\mathrm{TM}}$. In the present paper are presented the numerical aspects regarding the infusion of the resin in the preform undergoing compaction, and more specifically the numerical tools and schemes developed to deal with the resin region.

\section{Proposed model}

In the present paragraph is presented a brief summary of the main characteristics of the model developed to realize numerical simulations of infusion processes for organic composites. For details, the reader is invited to refer to Celle (2006) and Celle et al., (2008). A macroscopical approach is chosen for industrial use, based on a splitting of the resin-preform stacking in 3 zones representing both materials to mix, and the corresponding mixing: resin, preforms, saturated preforms (Figure 3). These zones are separated by moving boundaries and corresponding mechanical and thermal boundary conditions are defined (Celle et al., 2008). In this approach, macroscopically equivalent constitutive materials are considered, especially the preforms are represented as orthotropic porous media. This orthotropic feature may have an important influence regarding the preform compaction. Globally, more sophisticated equivalent constitutive laws can be introduced later when this model is validated.

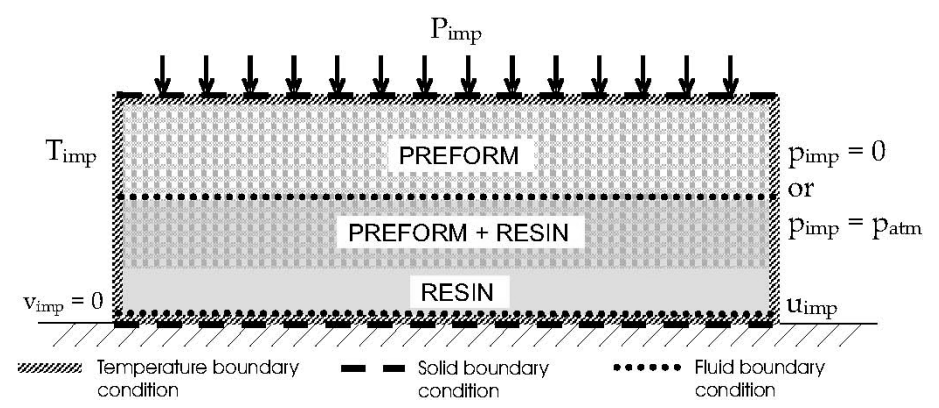

Figure 3. Splitting of the model geometry in 3 regions and associated boundary conditions

\subsection{Preform model}

The dry preform response is represented using an updated lagrangian formulation, specifically implemented in the existing eulerian based FE code (Celle, 
2006), associated with a non-linear elastic response under finite strains. Since the preform stacking geometry changes can be measured at every update and iteration, assuming that the fibre network is compressible but made up of incompressible fibres, the current fibre volume fraction can easily be calculated from the current transformation jacobian and these same parameters for the previous configuration:

$$
J(x, t+\Delta t)(1-\phi((x, t+\Delta t))=J(x, t)(1-\phi((x, t))
$$

where $J(x, t)$ is the jacobian of transformation at point $x$ and time $t$ and $\phi$ is the corresponding pore volume fraction. This possibility to calculate the current pore fraction (Equation 1) is one of the original features of the presented model, since only a global mechanical approach permits to access this parameter of great importance in infusion processes.

\subsection{Resin flow inside the preforms}

Regarding the resin flow, it is assumed to be laminar and inertial effects are neglected. The resin flow inside and outside the porous medium is modelled respectively through Darcy's (Darcy, 1856) and Stokes' equations in stationary regimes. The resin flow inside the porous medium accounts for the preforms deformation and for the resulting porosity variation during infusion, as explained earlier.

Representing the flow inside the porous medium depends essentially on the preform permeability. Indeed, one can figure out very easily that the ability of the resin to fill in the preforms will control the filling times, but also the filling level since the energy lost by the fluid to flow will equilibrate the energy brought in the system by the external pressure exerted. This permeability will strongly depend on the fibre volume fraction, i.e. on the preform compacity (Drapier et al., 2004), but also on its saturation (Bréard et al., 1999). For a given fluid velocity, permeability will control the fluid pressure, and conversely, the fluid pressure will modify the preform mechanical response due to its presence. To summarise, in the infusion processes studied here, the preform response will control the permeability through the corresponding fibre volume fraction, and hence the fluid pressure, while the fluid pressure induced in the pores will control the preform response due to its presence. Figure 4 represents a synthesis of the interactions between the preform mechanical behaviour and the resin flow.

Here, while reliable measurements are not available for longitudinal as well as for transverse permeabilities, and over a sufficiently wide range of fiber volume fractions (Drapier et al., 2002, 2004; Bréard et al., 1999), models will be considered. Regarding the preform mechanical response, in the fibrous saturated medium, a Terzaghi's model (Equation 2) permits to take into account the influence of the pore fluid pressure onto the preform response (Terzaghi, 1967; Kempner and Hahn, 1998; Gray and Schreffler, 2001 ). It writes in a eulerian form: 


$$
\sigma_{f}(x, t)=\sigma_{e f}(x, t)-s(x, t) p_{r}(x, t) \boldsymbol{I}
$$

where $\sigma_{f}$ is the Cauchy stress tensor in the "smeared-out" impregnated preform, $\sigma_{e f}$ is the Cauchy stress tensor in the preform "skeleton", $p_{r}$ is the resin pressure, $s$ is the saturation factor that may be taken into account if necessary, and $\boldsymbol{I}$ is the second order identity tensor.

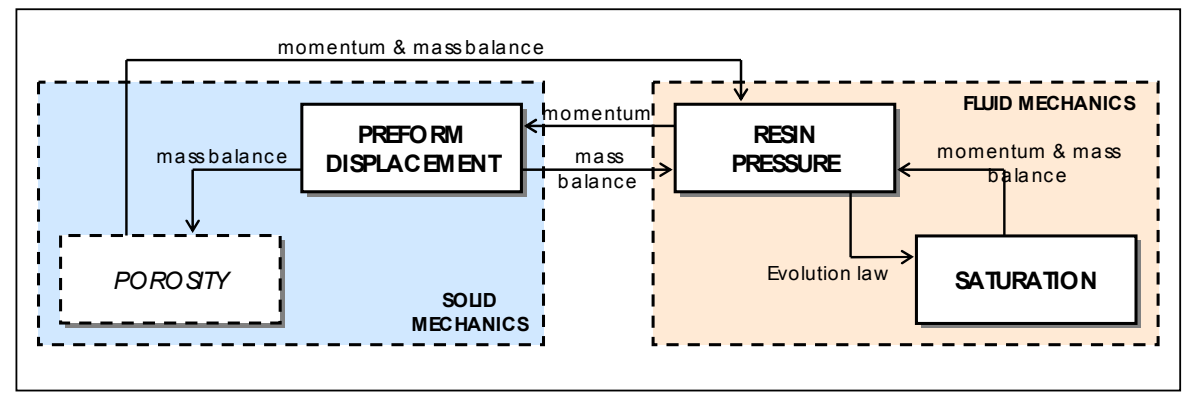

Figure 4. Synthesis of the interactions between the mechanical preform response and the resin flow in this porous medium

\subsection{Complementary couplings}

The previous interactions must be completed with continuity conditions at the mobile interfaces. These latter ensure both stress vector continuity and mass conservation. Between the saturated porous medium and the pure resin zone, they are more familiar as Beaver-Joseph-Shaffman conditions (Jäger and Mikelic, 2001). The tangential flow between these 2 zones is neglected in this presented first approach. Let us now focus on the pure resin region where the flow rises 3 major difficulties.

\section{Accounting for the pure resin region}

\subsection{Resin flow}

The incompressible fluid flow in the pure resin zone must be treated thanks to a particular formulation in order to satisfy the incompressibility, i.e. the mass balance. Here a mixed method is employed, based on the simultaneous resolution of the velocity and pressure fields in the resin using $\mathrm{P} 1+/ \mathrm{P} 1$ or $\mathrm{P} 1-\mathrm{buble} / \mathrm{P} 1$ finite elements. In order to satisfy the Brezzi Babuska stability condition (Arnold et al., 1984), this specific family of finite elements is considered. These elements are based on a further velocity degree of freedom, introduced at the centre of every element in the mesh. The shape function associated with this new degree of freedom equates 1 at 
the element centroid and vanishes on its edges. Then, the pressure and displacement velocity fields approximations write for a $n_{e}$ nodes finite element:

$$
\begin{aligned}
& p(\boldsymbol{x})=\Sigma_{1}^{n e} N_{i}(\boldsymbol{x}) p_{i} \\
& \{v(\boldsymbol{x})\}=\left\{v_{l}(\boldsymbol{x})\right\}+\left\{v_{b}(\boldsymbol{x})\right\}=\Sigma_{1}^{n e}\left[N_{i}(\boldsymbol{x})\right]\left\{v_{i}\right\}+N_{b}(\boldsymbol{x})\left\{v_{b}\right\}
\end{aligned}
$$

where the $N_{i}$ 's are the standard shape functions, $N_{b}$ and $p_{i},\left\{v_{i}\right\}$, and $\left\{v_{b}\right\}$ are respectively the nodal pressures, velocities and bubble velocity.

Figure 5 presents two examples of such bubble function. The polynomial bubble function which corresponds to the shape functions product for the linear element is the most basic. Arnold et al. (1984) show that the hierarchical function, equal to the minimum of the shape functions for the linear triangular element, yield excellent results even for coarse meshes. For some bubble functions that verify the orthogonality property, the elementary system to be solved for a linear element writes:

$$
\left[\begin{array}{cc}
\mathbf{K}_{r d}^{l} & \mathbf{K}_{i n}^{l^{T}} \\
\mathbf{K}_{i n}^{l} & \mathbf{C}
\end{array}\right]\left\{\begin{array}{l}
\mathbf{v} \\
p
\end{array}\right\}=\left\{\begin{array}{l}
\mathbf{F} \\
0
\end{array}\right\}
$$

where $\mathbf{K}_{r d}^{l}$ is the stiffness matrix associated with the linear velocity field, $\mathbf{K}_{i n}$ is the incompressibility matrix associated with the linear velocity field, $\mathbf{V}$ is a vector containing the element nodal velocities, $p$ contains the nodal pressures, $\mathbf{F}$ contains the external loadings and $\mathbf{C}$ is defined by $\mathbf{C}=\mathbf{K}_{i n}^{b} \cdot \mathbf{K}_{r d}^{b}{ }^{-1} \cdot \mathbf{K}_{i n}^{b^{T}}$ where $\mathbf{K}_{i n}^{b}$ et $\mathbf{K}_{r d}^{b}$ contain respectively incompressibility and stiffness matrices associated with the bubble velocity field.
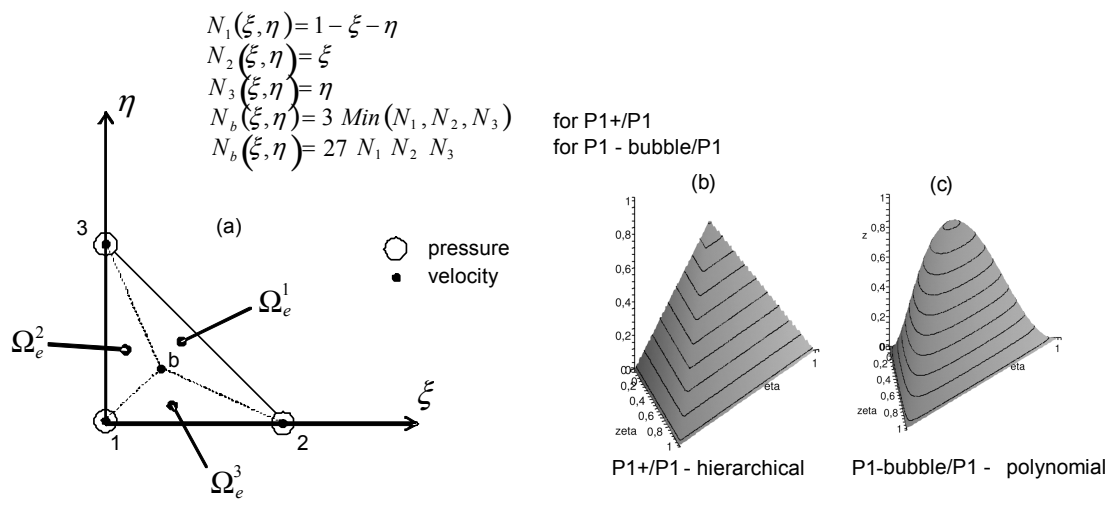

Figure 5. $P 1+/ P 1$ and $P 1 /$ buble Plfinite element - (a) discretisation, (b) Element $P 1+/ P 1$; hierarchical function and (c) P1-bubble/Plelement; polynomial function 
The bubble formulation employed in the treatment of the Stokes equations in stationary regime has also been used to deal with Darcy's equations in the saturated porous medium. Using the same approach permits to simplify the coupling between the Stokes and Darcy zones, and to propose the same method to solve for the flow in those two regions. Numerical results computed through these methods have been successfully validated by comparison with analytical results for test cases.

\subsection{Accounting for curvature effects}

2D sections and 3D models associated with composite pieces present usually curvature effects. These curvatures lead to zero normal velocity and displacements boundary conditions corresponding to impervious walls, that is to say absence of normal flow.

In order to take into account this type of condition, a penalty method has been first implemented in the eulerian FE code. This method permits to maintain the system size constant. Later, this method was improved by proposing a resolution of the kinematic condition directly in local frames associated with the nodes on boundaries. In this case, further operations are required for local/global referential switching. The resulting matrix system is however better conditionned and well suited to the use of iterative methods due to the disappearance of cross penalty terms.

\subsection{Remeshing}

Vanishing of the resin zone in the case of the RFI process must be represented. This leads to a major modification of the resin flow in the resin zone which must absolutely be integrated in order to obtain boundary conditions suitable to describe the flow in preforms. To deal with this disappearance, a remeshing technique is used. It is made up of three steps. The first step consists in defining a remeshing criterion $Q_{\text {mesh }}$. Here, the following angular criterion was chosen for triangular linear elements:

$$
Q_{\text {mesh }}=\min \left(Q_{e l t}\right)=\min \left(\frac{\alpha_{\min }}{\alpha_{\max }}\right)
$$

where $\alpha_{\min }$ and $\alpha_{\max }$ are respectively the smallest and largest angles of the considered element. The more the shape factor $Q_{\text {elt }}$ is close to 1 , the better its quality. The second step consists in the remeshing itself. To do so, the contour associated with the initial mesh is exported from the finite element libraries PROFLOT, remeshed in the free mesher $\mathrm{GMSH}^{\circledR}$ and then the new mesh is imported back into PRO-FLOT. The last step consists in transporting the nodal values from 
the old mesh into the new mesh. Every degree of freedom of the new mesh is projected in the old mesh and the element shape functions are used to recalculate the new nodal values.

\section{Few results}

An example of RFI simulation of a thin plate is presented in Figure 6. This highlights the main tools and formulations developed and implemented to deal with the resin zone and its vanishing. During the initial stage of compression, a high deformation of the preforms is observed (Figure 6-2), the corresponding pore volume fraction drops from 0.64 to 0.42 . When resin is sufficiently fluid, it begins to flow inside the preform network (Figure 6-3). As it can be verified in Figure 6-3, the presence of curvature leads to a non-homogeneous distribution of pressure in the resin film. Kinematical relationships between the degrees of freedom permit to model the absence of flow across the mould wall. During infusion, one can observe the progressive vanishing of the resin zone.

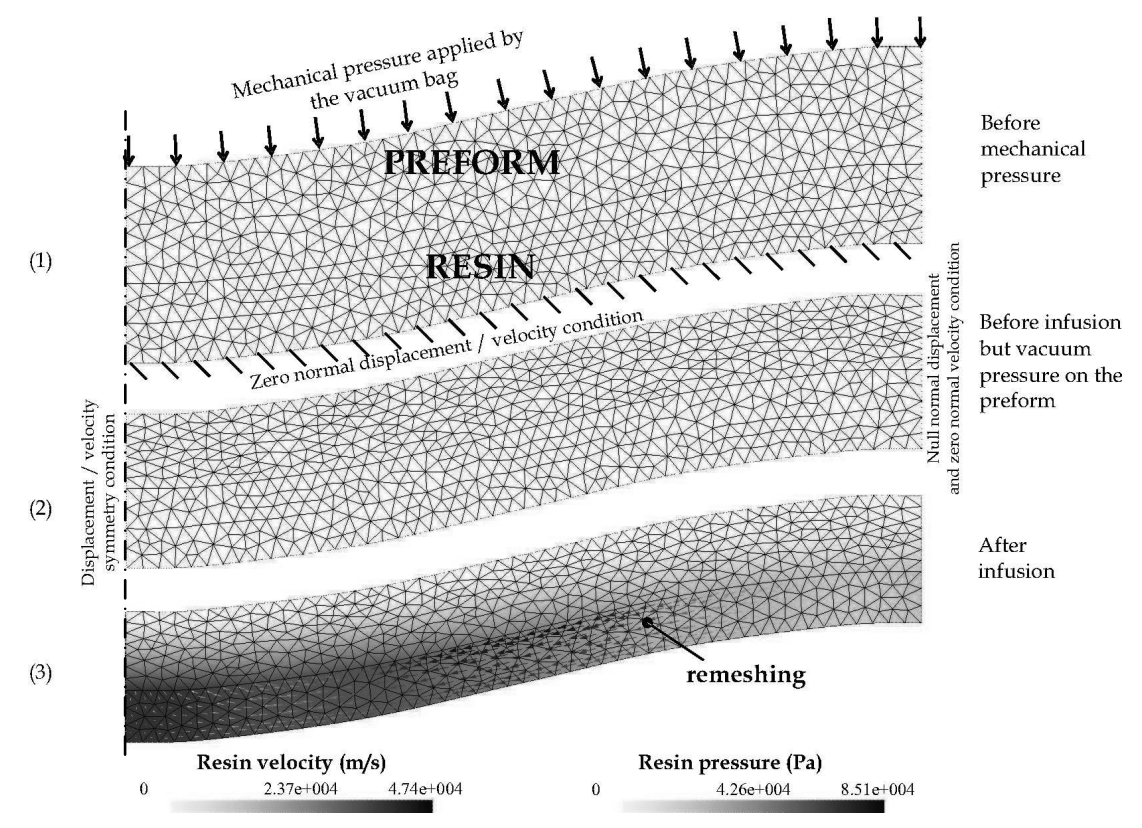

Figure 6. Numerical simulation of the infusion of a half curved plate elaborated with an infusion process: (1) Initial state, (2) Compaction, (3) Infusion 


\section{Conclusion}

A complete multi-physical model has been proposed to study infusion processes for composite materials (Celle, 2006 and Celle et al., 2008). Particularly, here were presented the numerical aspects relative to the resin zone treatment, and to its vanishing. This model can be adapted to various engineering fields, especially in biomechanics and geo-mechanics.

This work was achieved in the framework of a collaboration between ESI Group, Hexcel Corporation and Ecole Nationale Supérieure des Mines de Saint-Etienne.

\section{References}

Arnold D. N., Brezzi F., Fortin M., "A stable finite element for the Stokes equations", Estratto da Calcolo, vol. 21, 1984, p. 337-344.

Breard J., Henzel Y., Trochu F., "A standard characterization of saturated and unsaturated flow behaviors in porous media", Proceedings of the $12^{\text {th }}$ International Conference on Composite Materials ICCM12, Paris, 5-9 july 1999.

Celle P., Couplage fluide/milieu poreux en grandes déformations pour la modélisation des procédés d'élaboration des matériaux compostes par infusion, Thèse de doctorat de l'Ecole Nationale Supérieure des Mines de Saint-Etienne, 2006.

Celle P., Drapier S., Bergheau J.-M., "Numerical modeling of liquid infusion into fibrous media undergoing compaction", European Journal of Mechanics/A, vol. 27, n 4, 2008, p. 647-661.

Darcy H., Les fontaines publiques de la ville de Dijon, Dalmont, 1856.

Drapier S., Pagot A., Vautrin A., Henrat P., "Influence of the stitching density on the transverse permeability of Non-Crimped New Concept (NC2) multiaxial reinforcements: measurements and predictions", Composites Science and Technoogy, vol. 62, $\mathrm{n}^{\circ} 15$, 1979-1991, 2002.

Drapier S., Monnatte J., O. Elbouazzaoui, P. Henrat, "Characterization of transient through thickness permeabilities of Non Crimp New Concept (NC2) multiaxial fabrics", Composites/A, vol. 36, 2006, p. 877-892.

Gray W.G., Schrefler B. A., "Thermodynamic approach to effective stress in partially saturated porous media”, European Journal Mechanics, A/solids, 20, 2001, p. 521-538.

Jäger W., Mikelic A., "On the roughness-induced effective boundary conditions for an incompressible viscous flow", Journal of Differential Equations, vol. 170, n 1, p. 96-122, 2001.

Kempner E. A., Hahn H. T., "A unified approach to manufacturing simulation for composites", Proceedings of the First Korea-U.S. Workshop on Composite Materials, Seoul, Korea, 1998.

Terzaghi K., Peck R.B., Mesri G., Soil Mechanics in Engineering Practice, Jones Wiley \& Sons, New York, 1967. 
